\title{
Relay Enhanced LTE-Advanced Networks
}

\author{
Yousif Mohsin Hasan \\ University of Al-Qadisiyah, College of Engineering, Diwaniya 58002, Iraq
}

\begin{abstract}
Generation Project Partnership (3GPP) proposed the 4th generation mobile telecommunications (4G) technology [1], one such technology being Long-Term Evolution -Advanced (LTE-Advanced) to the ITU-T, which aims to improve the already existing Long Term Evolution (LTE-Release 8) technology. The need to provide high peak data rate to the meet of the demands of the market lead to enhancement of LTE-Release 8 which is LTE-A, that will provide an improved coverage range and increased bandwidth availability to end users. As LTE advanced is an enhanced version 8 rather than a total departure from LTE-Release 8. With LTEAdvanced having an increase in bandwidth of up to $100 \mathrm{MHz}$ that is achieved by means of carrier aggregation technique [2]. The main aim of this paper is to provide a detailed overview of relay concepts in LTE technology and discuss the advancement from LTE towards LTE-Advanced technology with references to LTE supporting technologies such as Orthogonal Frequency-Division Multiplexing (OFDM), Coordinated Multi-Point transmission (CoMP), Multiple-Input Multiple-Output (MIMO) and Carrier Aggression and issues involved in its implementations.
\end{abstract}

Keywords: LTE-Release 8, LTE-Advanced, ITU-T, 3rd GPP, Relay, OFDM, CoMP, MIMO, Carrier Aggression

\section{Introduction}

The high bandwidth required in the new wireless communication era has necessitated the need for a more cost efficient way to extend network coverage and enhancement in the throughput. LTE-Release 8 technology employs a macro and micro cell design architecture, provides a high peak-data rate of up to $300 \mathrm{Mb} / \mathrm{s}$ at the $\mathrm{DL}$ and $75 \mathrm{Mb} / \mathrm{s}$ on the UL for a bandwidth of up to $20 \mathrm{MHz}$, less operation cost, support for multi-antenna system and flexibility operational bandwidth [3].An overall system capacity as well as increasing the area/region of coverage and a transparent integration of already existing technology or system(s). The Enhancement of LTE-Release 8 is LTE-Release 10 also known as LTE-Advanced was proposed by 3rd GPP which seeks to provide a structure frame-work (support low power consuming nodes which comprise of relays, pico/ femto cells and much more), the UL of support data rate of up-to $500 \mathrm{Mb} / \mathrm{s}$ with a spectral efficiency of $15 \mathrm{HZ}$ and a DL of data rate of up-to $1 \mathrm{~Gb} / \mathrm{s}$ with spectral efficiency of about $30 \mathrm{~b} / \mathrm{s}$ which satisfies the requirement of International Mobile Telecommunications- Advanced (IMT-Advanced) as set by International Telecommunication Union-Radio communication (ITU-R). However this additional feature to include in LTE-Advanced leads to a lot of complexities and difficulties in implementation.

This paper describes the basics of relaying concepts, overview of 3GPP LTE-Release 8, enhanced specifications of LTE-Advanced technology along with its supporting technologies for instance Orthogonal Frequency-Division Multiplexing (OFDM), Coordinated Multi-Point transmission (CoMP), Multiple Input Multiple Output (MIMO) and Carrier Aggregation.

\section{Related Works}

LTE-Advanced is the enhancement of LTE-Release 8 [4] as increased features are added to LTE-Advanced to provide and increased performance of the LTE technology, with LTE-Advance not been a total depart from LTE Release but an improved or enhanced version[6]. Recently various researches have been done in this area to achieve costefficiency, coverage expansion and enhancement in throughput for LTE-Advanced network [7]. Mostly the research focused on relay performances analysis [8], while others placing more emphasis on the architecture of the relay to minimize cost of implementation along with the use of relay nodes $(\mathrm{RN})$ to increase network coverage [9].

\section{Basic Concepts of Relay Network Architecture}

Relay system (LTE -Advanced) is a technology that provides extension of coverage, capacity and enables flexibility deployment at low cost. A relay system can operate in both, in-band or out-band transmission modes [4]. It consists of Relay Node (RN) and evolved Node B (eNB). Relay system architecture can be summarized as follows;

- Relays are used to cut the distance between the eNB and user equipment (UE) which could be either in a transparent or un-transparent manner. In the transparent manner the UE is un-aware of the presence of the relay node while the un-transparent manner the user equipment is aware of the presence of a relay node.

- The relay cells can be synchronized to a relay node (RN) through a cell search directly.

- In-band or out-band transmission modes are supported between the eNB and the RN. In the in band transmission a single spectrum is shared between the access link and the backhaul link.

- A UE can be only be contacted to a donor eNB or a RN one at a time.

- In a single-cell operation, information and HARQ feedback are received by the UE through a RN as it sends a control-channel to the $\mathrm{RN}$.

- In a backward compatibility, UEs can be served by RN. 


\section{International Journal of Science and Research (IJSR) \\ ISSN (Online): 2319-7064}

Index Copernicus Value (2013): 6.14 | Impact Factor (2014): 5.611
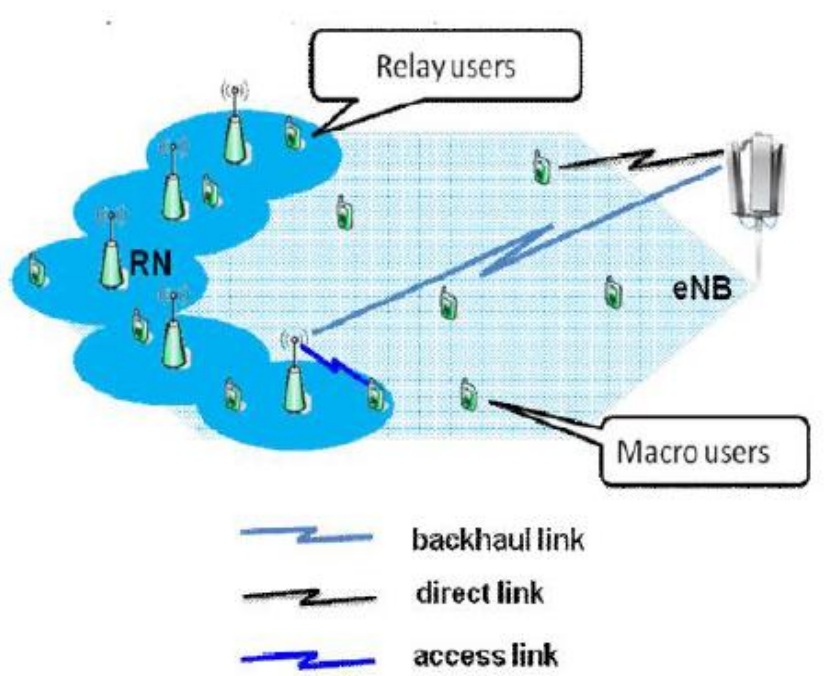

Figure 1: The pictorial explanation of a Relay Network

\section{LTE-Release 8}

LTE-release 8 employs an orthogonal frequency-division multiplexing (OFDM) at the downlink while at uplink a single carrier frequency-division multiple-access (SCFDMA) and an optimized system-architecture evolution (SAE) is employed.

As LTE-Release 8 employs a DL/UL at selective frequency and diverse frequency schedule, sub-frame structure of the DL is common to time division-duplex (TDD) and frequency division-duplex (FDD)[1]. LTE-Release 8 provides high performance at air-interface which allows higher data-rates to users at low-latency. At the Uplink a single-carrier frequency division-multiplexing (SC-FDM) is implemented easily via a Discrete Fourier Transform Spread OFDM (DFT-S-OFDM), while downlink employing an OFDM scheme of transmission. OFDM transmission-scheme however provides improvement in the transmitted power, cell-edge coverage and a minimum interference at the eNB [7]. The use of Orthogonal Frequency Division Multiple Access (OFDMA) provides a flexible spectrum. As DFT-SOFDM with a similar but different numerology to the downlink, OFDM transmission scheme with different DFT constellation symbols are pre-coded before being mapped at different sub-carriers. As DFT per-code tends to improve the maximum power transmitted and cell coverage is greatly improved to allow a longer battery life.

\section{LTE-Advanced}

LTE-Advanced is an enhanced version of LTE-Release 8, as LTE-Advanced design frame work is aimed at satisfying the requirements of IMT-A to which LTE-Release 8 does not satisfy [2]. As ITE-A is a mobile system that provides wide varieties for telecommunication services such as advanced mobile services, been supported by mobile as well as fixed networks and are packet-based.

The requirement to which LTE-Advanced aims to satisfy and meet IMT-A requirements are summarized below [5]. Employing of carrier aggregation, spatial-multiplexing using as much as 8 layers multiple input-multiple-output (MIMO) and inter-cell CoMP at transmission and reception downlink and also uplink spatial-multiplexing which employs a four layered MIMO. Latency is reduced to improve the spectralefficiency which achieved through reduction of the of connected from idle to $50 \mathrm{mseconds}$ in LTE-Advanced as LTE-Release 8 is at $100 \mathrm{mseconds}$ and a transition time from dormant to active is to be reduced from 50 mseconds in LTERelease 8 to 10 miliseconds in LTE-Advanced [2].Enhanced capacity and increased coverage is achieved in LTEAdvanced via a heterogeneous-network which is made up of low-power consuming nodes to provide an improved system output.

Table 1:Comparison between LTE-Release 8 and LTE-

\begin{tabular}{|c|c|c|c|}
\hline \multicolumn{4}{|c|}{ Advanced } \\
\hline & & LTE Release 8 & LTE-Advanced \\
\hline \multirow[t]{2}{*}{ Peak data rate } & Downlink & $300 \mathrm{Mbps}$ & $1 \mathrm{Gbps}$ \\
\hline & Uplink & $75 \mathrm{Mbps}$ & $500 \mathrm{Mbps}$ \\
\hline \multirow{2}{*}{$\begin{array}{c}\text { Peak spectrum } \\
\text { efficiency } \\
\text { [bps/Hz] }\end{array}$} & Downlink & $\begin{array}{c}15 \\
4 * 4 \text { MIMO }\end{array}$ & $\begin{array}{c}30 \\
\text { Up to } 8 * 8 \text { MIMO }\end{array}$ \\
\hline & Uplink & $\begin{array}{c}3.75 \\
\text { 64QAM SISO }\end{array}$ & $\begin{array}{c}15 \\
\text { Up to } 4 * 4 \text { MIMO }\end{array}$ \\
\hline \multirow{2}{*}{$\begin{array}{c}\text { Transmission } \\
\text { Band-width } \\
\end{array}$} & Downlink & $20 \mathrm{Mbps}$ & $100 \mathrm{Mbps}$ \\
\hline & Uplink & $20 \mathrm{Mbps}$ & $40 \mathrm{Mbps}$ \\
\hline \multicolumn{2}{|c|}{ Scalable Band-width } & $1.4-20 \mathrm{MHz}$ & $20-100 \mathrm{MHz}$ \\
\hline
\end{tabular}

\section{Carrier Aggregation}

With bandwidth increase achievable of up to $100 \mathrm{MHz}$ in LTE-Advanced via a carrier Aggregation. Carrier aggregation enables data peak rates in an excess of $1 \mathrm{~GB} / \mathrm{s}$ at the DL and $500 \mathrm{Mb} / \mathrm{s}$ at the UL [3]. Carrier aggregation permits for operators to operate systems that have an extended band-width which is achieved by means of aggregation of several smaller component carriers when providing of backward compatibility for end users.

Three types of carrier components can visualized as follows:

- Back-ward compatible carrier: with all LTE-Advance UE been able to access the carrier type regardless the release version. As all features of LTE are be supported.

- Non Back-ward compatible carrier: As only LTE-A is compatible this carrier, while supporting advanced features like control less-operations which primary not formed in LTE-Release UE.

- Extension carrier: As the carrier type is used to extend another type of carrier, it can be applied where high interference is present at the macro, provide service to home eNBs. With access of extension carrier only provide as a part of a carrier aggregation set as it uses a PDCCH and a separate HARQ process.

Possible scenarios of carrier Aggregation are as follows a contiguous-aggregation of carrier component in a singleband, non-contiguous aggregation of carrier component in single or over multiple bands. 


\section{International Journal of Science and Research (IJSR)}

ISSN (Online): 2319-7064

Index Copernicus Value (2013): 6.14 | Impact Factor (2014): 5.611

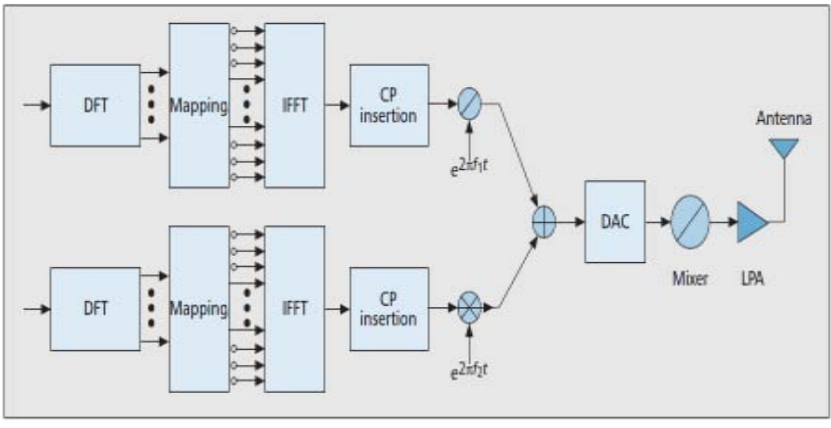

Figure 2: Block diagram of an uplink carrier aggregation for a transmitter.

\section{MIMO Enhancements for LTE-Advanced}

Multiple-input multiple-output feature is critically in LTEAdvanced meeting to its objective of a multiple-antenna technology. With characteristics of beam formation and spatial-multiplexing, which have featured prominently in LTE-A.

To improve overall system efficiency, enhancements are made in MIMO technologies used in LTE-Advanced [8]. LTE-Advanced has an antenna configuration of 8 by 8 at the downlink and a 4 by 4 at the uplink.

\section{Downlink MIMO Transmission}

Spatial multiplexing scheme is enhanced in 8 layered transmissions with an improved signal-reference structure in LTE-Advance. With the signal-reference overhead not be proportional to the value of instantaneous transmission-rank rather the value of the maximum-transmission supported rank obtained when relaying in a high ordered spatial multiplexing on a cell specific-reference. As UE specificreference signal supports as much up to 8 layered demodulations in LTE-Advanced.

\section{Coordinated Multipoint Transmission}

A common dominator factor dynamic coordinated of transmission / reception at separated multiple-geographically sites which are aimed at improving the system efficiency and the quality-of-service for end users [7]. CoMP comprises of various coordinated schemes (dynamic inter-cell scheduling co-ordination to joint transmission/reception at multiplesites).With signals from multi-sites processed jointly an improvement can be achieved that the reception this technique is the Joint reception. The combination of the uplink received transmissions at multi-points can be like interference rejection-combination and a max-ratio combination with some similarities to the softer hand-over employed at sites.

\section{Results and Discussion}

The paper highlights the technology swift in LTE-Release 8technology and the tremendous benefits it has over the existing technology. However demand for higher bandwidth to meet the needs of modern mobile communication which has necessitated the drive to enhance LTE-Release 8 even more which has led to the proposal of LTE-Advanced. The
Table 1 gives a comparison between LTE-Release 8 and LTE-Advanced.

However LTE-Advanced technology brings about a lot of complexities and difficulties in its implementation [10].

Implementation of new features requires for alterations, in the network architecture.

Relaying techniques can enhance expansion network coverage but the concept of Relaying in LTE-Advanced has not been included in the specification of LTE-Advance yet.

\section{Conclusion and Recommendations for Future Work}

The enhancement of LTE-Release 8 is the brain behind the LTE-Advanced project as it aims to provide a higher datarate, bandwidth and capacity to the user. This paper has explained some features that are to be supported by the proposed LTE-Advanced system which are OFDM, MIMO, CoMP and Carrier Aggregation. However, as LTE-Advanced still remains an ongoing project, further features are expected to be added to it as for instance the use of non-transparent relays that are also known as type 1 relays which will allow the relay to maintain its own identity and broadcast control signaling to other user equipment. Moreover the scheduling can also be done independently in the relay node.

\section{References}

[1] Barbieri, A., Gaal, P., Geirhofer, S., Ji, T., Malladi, D. Wei, Y., \&Xue, F. (2012, February). Coordinated downlink multi-point communications in heterogeneous cellular networks. In Information Theory and Applications Workshop (ITA), 2012 (pp. 7-16). IEEE.

[2] Huang, X., Ulupinar, F., Agashe, P., Ho, D., \&Bao, G. (2010, December). LTE relay architecture and its upper layer solutions. In Global Telecommunications Conference (GLOBECOM 2010), 2010 IEEE (pp. 1-6). IEEE.

[3] Irmer, R., \&Diehm, F. (2008, September). On coverage and capacity of relaying in LTE-advanced in example deployments. In Personal, Indoor and Mobile Radio Communications, 2008. PIMRC 2008. IEEE 19th International Symposium on (pp. 1-5). IEEE.

[4] Parkvall, S., Dahlman, E., Furuskar, A., Jading, Y., Olsson, M., Wänstedt, S., \&Zangi, K. C. (2008, September). LTE-Advanced-Evolving LTE towards IMT-Advanced. In VTC Fall (pp. 1-5).

[5] Rong, L., Elayoubi, S. E., \&Haddada, O. B. (2010, May). Impact of relays on LTE-Advanced performance. In Communications (ICC), 2010 IEEE International Conference on (pp. 1-6). IEEE.

[6] Saleh, A. B., Redana, S., Raaf, B., Riihonen, T. Hämäläinen, J., \&Wichman, R. (2009, September). Performance of amplify-and-forward and decode-andforward relays in LTE-advanced. In Vehicular Technology Conference Fall (VTC 2009-Fall), 2009 IEEE 70th (pp. 1-5). IEEE.

[7] Sawahashi, M., Kishiyama, Y., Morimoto, A., Nishikawa, D., \&Tanno, M. (2010). Coordinated 


\section{International Journal of Science and Research (IJSR) \\ ISSN (Online): 2319-7064}

Index Copernicus Value (2013): 6.14 | Impact Factor (2014): 5.611

multipoint transmission/reception techniques for LTEadvanced [Coordinated and Distributed MIMO]. Wireless Communications, IEEE, 17(3), 2634.

[8] Scheme, B. T. (2009). LTE: the evolution of mobile broadband. IEEE Communications magazine, 45 .

[9] Series, M. (2009). Guidelines for evaluation of radio interface technologies for IMT-Advanced. Report ITU, 2135-1?

[10] Yang, Y., Hu, H., Xu, J., \& Mao, G. (2009). Relay technologies for WiMAX and LTE-advanced mobile systems. Communications Magazine, IEEE, 47(10), 100-105.

\section{Author Profile}

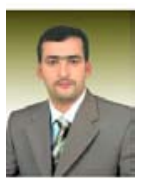

Yousif Mohsin Hasan receivedB.Sc. degree inElectronic Engineering, university of Mosul, Mosul, Iraq, 2006 and he received M.Sc. degree in Electrical engineering/ Electronics and Telecommunications, UTM, Johor, Malaysia, 2015. He has Experience as Engineer in University of Al- Al-Qadisiyah/ College of Engineering since 2007 and Lecturer in University of Al- Al-Qadisiyah/ College of Engineering since 2015.he is teaching. Electrical Engineering and control engineering.Published papers

1. Link Budget Analysis for Underwater Communication System

2. Compact Multiband MIMO Antenna for Future Wireless Applications 